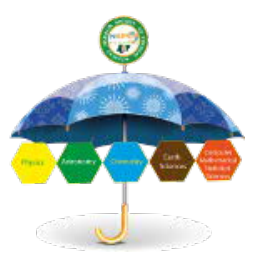

\title{
Common Fixed Point Theorems for Multivalued Generalized $F$-Suzuki-Contraction Mappings in Complete Strong $b$-Metric Spaces
}

\author{
Yusuf Ibrahim* \\ Department of Mathematics, Sa'adatu Rimi College of Education, Kumbotso Kano, Nigeria
}

\begin{abstract}
This paper introduces a new version of multivalued generalized $F$-Suzuki-Contraction mapping and then establish some new common fixed point theorems for these new multivalued generalized $F$-Suzuki-Contraction Mappings in complete strong $b$-Metric Spaces.
\end{abstract}

Keywords: Common Fixed Point Problem, Multivalued Generalized $F$-Suzuki-Contraction Mapping, Complete Strong $b$-metric Space.

Article History:

Received: 04 April 2019

Received in revised form: 01 September 2019

Accepted for publication: 02 September 2019

Published: 28 September 2019

(c)2019 Journal of the Nigerian Society of Physical Sciences. All Rights Reserved.

Communicated by: B. J. Falaye

\section{Introduction}

Let $X$ be a nonempty set and $s \geq 1$ be a given real number. A mapping $d: X \times X \rightarrow \mathbb{R}^{*}$ is said to be a $b$-metric if for all $x, y, z \in X$ the following conditions are satisfied:

1. $d(x, y)=0$ if and only if $x=y$;

2. $d(x, y)=d(y, x)$;

3. $d(x, z) \leq s[d(x, y)+d(y, z)]$.

The pair $(X, d)$ is called a $b$-metric space with constant $s$. A strong $b$-metric is a semimetric space $(X, d)$ if there exists $s \geq 1$ for which $d$ satisfies the following triangular inequality.

$$
d(x, y) \leq d(x, z)+\operatorname{sd}(z, y), \text { for each } x, y, z \in X .
$$

In 1922, a mathematician Banach [1] proved a very important result regarding a contraction mapping, known as the Banach contraction principle, which states that every self-mapping $T$ defined on a complete metric space $(X, d)$ satisfying

$$
\forall x, y \in X, d(T x, T y) \leq \lambda d(x, y), \text { where } \lambda \in(0,1)
$$

has a unique fixed point and for every $x_{0} \in X$ a sequence $\left\{T_{n} x_{0}\right\}_{n=1}^{\infty}$ converges to the fixed point. Subsequently, in 1962, Edelstein [2] proved the following version of the Banach contraction principle. Let $(X, d)$ be a compact metric space and let $T: X \rightarrow X$ be a self-mapping. Assume that for all $x, y \in X$ with $x \neq y$,

$$
d(x, T x)<d(x, y) \Longrightarrow d(T x, T y)<d(x, y) .
$$

Then $T$ has a unique fixed point in $X$. In 2012, Wardowski [3] introduced a new type of contractions called F-contraction and proved a new fixed point theorem concerning F-contractions.

Let $(X, d)$ be a metric space. A mapping $T: X \rightarrow X$ is said to be an F-contraction if there exists $\tau>0$ such that

$$
\forall x, y \in X, d(T x, T y)>0 \Longrightarrow \tau+F(d(T x, T y)) \leq F(d(x, y)),
$$


where $F: R^{+} \rightarrow R$ is a mapping satisfying the following conditions:

F1 $\mathrm{F}$ is strictly increasing, i.e. for all $x, y \in R^{+}$such that $x<y, F(x)<F(y)$;

F2 For each sequence $\left\{\alpha_{n}\right\}_{n=1}^{\infty}$ of positive numbers, $\lim _{n \rightarrow \infty} \alpha_{n}=$ 0 if and only if $\lim _{n \rightarrow \infty} F\left(\alpha_{n}\right)=-\infty$;

F3 There exists $k \in(0,1)$ such that $\lim _{\alpha \rightarrow 0^{+}} \alpha^{k} F(\alpha)=0$.

We denote by $\zeta$, the set of all functions satisfying the conditions $(F 1)-(F 3)$. Wardowski [3] then stated a modified version of the Banach contraction principle as follows. Let $(X, d)$ be a complete metric space and let $T: X \rightarrow X$ be an F-contraction. Then $T$ has a unique fixed point $x^{*} \in X$ and for every $x \in X$ the sequence $\left\{T_{n} x\right\}_{n=1}^{\infty}$ converges to $x^{*}$. In 2014, Hossein, P. and Poom, K. [15] defined the F-Suzuki contraction as follows and gave another version of theorem. Let $(X, d)$ be a metric space. A mapping $T: X \rightarrow X$ is said to be an F-Suzuki-contraction if there exists $\tau>0$ such that for all $x, y \in X$ with $T x \neq T y$

$$
d(x, T x)<d(x, y) \Longrightarrow \tau+F(d(T x, T y)) \leq F(d(x, y)),
$$

where $F: R^{+} \rightarrow R$ is a mapping satisfying the following conditions:

$\mathrm{F} 1 \mathrm{~F}$ is strictly increasing, i.e. for all $x, y \in R^{+}$such that $x<y, F(x)<F(y)$;

F2 For each sequence $\left\{\alpha_{n}\right\}_{n=1}^{\infty}$ of positive numbers, $\lim _{n \rightarrow \infty} \alpha_{n}=$ 0 if and only if $\lim _{n \rightarrow \infty} F\left(\alpha_{n}\right)=-\infty$;

F3 $F$ is continuous on $(0, \infty)$

We denote by $\zeta$, the set of all functions satisfying the conditions $(F 1)-(F 3)$.

Let $T$ be a self-mapping of a complete metric space $X$ into itself. Suppose $F \in \zeta$ and there exists $\tau>0$ such that

$$
\forall x, y \in X, d(T x, T y)>0 \Longrightarrow \tau+F(d(T x, T y)) \leq F(d(x, y)) .
$$

Then $T$ has a unique fixed point $x^{*} \in X$ and for every $x_{0} \in X$ the sequence $\left\{T_{n} x_{0}\right\}_{n=1}^{\infty}$ converges to $x^{*}$.

Following this direction of research (see examples, [4, 5, 6, 7, $8,9,10,16,17])$, in this paper, fixed point results of Piri and Kumam [11], Ahmad et al. [9], Suzuki [18] and Suzuki [19] are extended by introducing common fixed point problem for multivalued generalized F-Suzuki-contraction mappings in strong b-metric spaces.

\section{Definition 1.1. (Hardy and Rogers [14])}

(1) There exist non-negative constants $a$, satisfying $\sum_{i=1}^{5} a_{i}<$ 1 such that, for each $x, y \in X, d(f(x), f(y))<a_{1} d(x, y)+$ $a_{2} d(x, f(x))+a_{3} d(y, f(y))+a_{4} d(x, f(y))+a_{5} d(y, f(x))$.

(2) There exist monotonically decreasing functions $a_{i}(t)$ : $(0, \infty) \rightarrow[0,1)$ satisfying $\sum_{i=1}^{5} a_{i}(t)<1$ such that, for each $x, y \in X, x \neq y, d(f(x), f(y))<a_{1}(d(x, y)) d(x, f(x))$ $+a_{2}(d(x, y)) d(y, f(y))+a_{3}(d(x, y)) d(x, f(y))$ $+a_{4}(d(x, y)) d(y, f(x))+a_{5}(d(x, y)) d(x, y)$.
(3) For each $x, y \in X, x \neq y$, $d(f(x), f(y))<\max \{d(x, y), d(x, f(x))$, $d(y, f(y)), d(x, f(y)), d(y, f(x))\}$.

Lemma 1.1. [13] From definition 1.1, (1) $\Longrightarrow(2) \Longrightarrow(3)$.

Denote by $C B(X)$, the collection of all nonempty closed and bounded subsets of $X$ and let $H$ be the Hausdorff metric with respect to the metric $d$; that is,

$$
H(A, B)=\max \left\{\sup _{a \in A} d(a, B), \sup _{b \in B} d(b, A)\right\}
$$

for all $A, B \in C B(X)$, where $d(a, B)=\inf _{b \in B} d(a, b)$ is the distance from the point $a$ to the subset $B$.

\section{Main Results}

Definition 2.1. Let $\mho$ be the family of all functions $F: R^{+} \rightarrow R$ such that:

(F1) $F$ is strictly increasing, i.e. for all $x, y \in R^{+}$such that $x<y, F(x)<F(y)$;

(F2) for each sequence $\left\{\alpha_{n}\right\}_{n=1}^{\infty}$ of positive numbers, $\lim _{n \rightarrow \infty} \alpha_{n}=$ 0 if and only if $\lim _{n \rightarrow \infty} F\left(\alpha_{n}\right)=-\infty$;

(F3) $F$ is continuous on $(0, \infty)$.

Definition 2.2. Let $\Psi$ be the family of all functions $\psi:[0, \infty) \rightarrow$ $[0, \infty)$ such that $\psi$ is continuous and $\psi(t)=0$ iff $t=0$.

Definition 2.3. Let $(X, d)$ be a strong $b$-metric space. Mappings $T, S: X \rightarrow C B(X)$ are said to be multivalued generalized $F$-Suzuki-Contraction on $(X, d)$ if there exists $F \in \mho$ and $\psi \in \Psi$ such that, $\forall x, y \in X, x \neq y$,

$$
\frac{1}{1+s} d(x, T x)<d(x, y) \text { and } \frac{1}{1+s} d(y, S y)<d(y, S T x)
$$

$\Rightarrow \psi\left(N_{\phi}(x, y)\right)+F\left(s^{4} H(T x, S y)\right) \leq F\left(N_{\phi}(x, y)\right)$ in which

$$
\begin{aligned}
& N_{\phi}(x, y)=\phi_{1}(d(x, y))(d(x, y))+\phi_{2}(d(x, y))(d(y, S T x)) \\
& +\phi_{3}(d(x, y))\left(\frac{(d(y, T x))+d(x, S y)}{2 s}\right) \\
& +\phi_{4}(d(x, y))\left(\frac{(d(x, S T x))+H(S T x, S y)}{2 s}\right) \\
& +\phi_{5}(d(x, y))(H(S T x, S y)+H(S T x, T x)) \\
& +\phi_{6}(d(x, y))(H(S T x, S y)+d(T x, x)) \\
& \left.+\phi_{7}(d(x, y))(d(T x, y))+d(y, S y)\right)
\end{aligned}
$$

for which $\phi: R^{+} \rightarrow[0,1)$, with $\sum_{i=1}^{7} \phi_{i}(d(x, y))<1$, is monotonically decreasing function.

Comsidering the definition $S T x:=\{S y \subseteq C B(X): \forall y \in T x\}$, we have the following result.

Theorem 2.1. Let $(X, d)$ be a complete strong $b$-metric space and let $T, S: X \rightarrow C B(X)$ be multivalued generalized $F$ Suzuki-Contraction mappings. Then $T$ and $S$ has a common 
fixed point $x^{*} \in X$ and for every $x \in X$ the sequence $\left\{T^{n} x\right\}_{n}^{\infty}$ and $\left\{S^{n} x\right\}_{n}^{\infty}$ converge to $x^{*}$.

Proof Let $x_{0}=x \in X$. Let $x_{n+1} \in T x_{n}$ and $x_{n+2} \in S x_{n+1} \forall n \in$ $N$. If there exists $n \in N$ such that $d\left(x_{n}, T x_{n}\right)=d\left(x_{n+1}, S x_{n+1}\right)=$ 0 then $x_{n+1}=x_{n}=x$ becomes a fixed point of $T$ and $S$, respectively, therefore the proof is complete. Now, suppose that $d\left(x_{n}, T x_{n}\right)>0$ and $d\left(x_{n+1}, S x_{n+1}\right)>0 \forall n \in N$ then the proof will be divided in to two steps.

Step one. We show that $\left\{x_{n}\right\}_{n=1}^{\infty}$ is a Cauchy sequence.

Let

$$
d\left(x_{n}, T x_{n}\right)>0 \text { and } d\left(x_{n+1}, S x_{n+1}\right)>0 \forall n \in N
$$

therefore, we have that

$$
\begin{aligned}
& \frac{1}{s+1} d\left(x_{n}, T x_{n}\right)<d\left(x_{n}, T x_{n}\right) \text { and } \\
& \frac{1}{s+1} d\left(x_{n+1}, S x_{n+1}\right)<d\left(x_{n+1}, S x_{n+1}\right) \forall n \in N .
\end{aligned}
$$

By Definition 2.3, we get

$$
F\left(H\left(T x_{n}, S x_{n+1}\right)\right) \leq F\left(N_{\phi}\left(x_{n}, x_{n+1}\right)\right)-\psi\left(N_{\phi}\left(x_{n}, x_{n+1}\right)\right) .
$$

Since that

$$
\begin{aligned}
& N_{\phi}\left(x_{n}, x_{n+1}\right) \\
& =\phi_{1}\left(d\left(x_{n}, x_{n+1}\right)\right)\left(d\left(x_{n}, x_{n+1}\right)\right)+\phi_{2}\left(d\left(x_{n}, x_{n+1}\right)\right)\left(d\left(x_{n+1}, x_{n+2}\right)\right) \\
& +\phi_{3}\left(d\left(x_{n}, x_{n+1}\right)\right)\left(\frac{d\left(x_{n}, x_{n+2}\right)}{2 s}\right)+\phi_{4}\left(d\left(x_{n}, x_{n+1}\right)\right)\left(\frac{\left(d\left(x_{n}, x_{n+2}\right)\right)}{2 s}\right) \\
& +\phi_{5}\left(d\left(x_{n}, x_{n+1}\right)\right)\left(d\left(x_{n+2}, x_{n+1}\right)\right)+\phi_{6}\left(d\left(x_{n}, x_{n+1}\right)\right)\left(d\left(x_{n}, x_{n+1}\right)\right) \\
& +\phi_{7}\left(d\left(x_{n}, x_{n+1}\right)\right)\left(d\left(x_{n+2}, x_{n+1}\right)\right. \\
& \leq \phi_{1}\left(d\left(x_{n}, x_{n+1}\right)\right)\left(d\left(x_{n}, x_{n+1}\right)\right)+\phi_{2}\left(d\left(x_{n}, x_{n+1}\right)\right)\left(d\left(x_{n+1}, x_{n+2}\right)\right) \\
& +\phi_{3}\left(d\left(x_{n}, x_{n+1}\right)\right)\left(\frac{d\left(x_{n}, x_{n+1}\right)+s d\left(x_{n+1}, x_{n+2}\right)}{2 s}\right) \\
& +\phi_{4}\left(d\left(x_{n}, x_{n+1}\right)\right)\left(\frac{d\left(x_{n}, x_{n+1}\right)+s d\left(x_{n+1}, x_{n+2}\right)}{2 s}\right) \\
& +\phi_{5}\left(d\left(x_{n}, x_{n+1}\right)\right)\left(d\left(x_{n+2}, x_{n+1}\right)\right)+\phi_{6}\left(d\left(x_{n}, x_{n+1}\right)\right)\left(d\left(x_{n}, x_{n+1}\right)\right) \\
& +\phi_{7}\left(d\left(x_{n}, x_{n+1}\right)\right)\left(d\left(x_{n+2}, x_{n+1}\right)\right. \\
& \leq \phi_{1}\left(d\left(x_{n}, x_{n+1}\right)\right)\left(d\left(x_{n}, x_{n+1}\right)\right)+\phi_{2}\left(d\left(x_{n}, x_{n+1}\right)\right)\left(d\left(x_{n+1}, x_{n+2}\right)\right) \\
& +\phi_{3}\left(d\left(x_{n}, x_{n+1}\right)\right)\left(\frac{s\left[d\left(x_{n}, x_{n+1}\right)+d\left(x_{n+1}, x_{n+2}\right)\right]}{2 s}\right) \\
& +\phi_{4}\left(d\left(x_{n}, x_{n+1}\right)\right)\left(\frac{s\left[d\left(x_{n}, x_{n+1}\right)+d\left(x_{n+1}, x_{n+2}\right)\right]}{2 s}\right) \\
& +\phi_{5}\left(d\left(x_{n}, x_{n+1}\right)\right)\left(d\left(x_{n+2}, x_{n+1}\right)\right)+\phi_{6}\left(d\left(x_{n}, x_{n+1}\right)\right)\left(d\left(x_{n}, x_{n+1}\right)\right) \\
& +\phi_{7}\left(d\left(x_{n}, x_{n+1}\right)\right)\left(d\left(x_{n+2}, x_{n+1}\right)\right. \\
& \leq \phi_{1}\left(d\left(x_{n}, x_{n+1}\right)\right)\left(d\left(x_{n}, x_{n+1}\right)\right)+\phi_{2}\left(d\left(x_{n}, x_{n+1}\right)\right)\left(d\left(x_{n+1}, x_{n+2}\right)\right) \\
& +\phi_{3}\left(d\left(x_{n}, x_{n+1}\right)\right)\left(d\left(x_{n}, x_{n+1}\right)\right)+\phi_{3}\left(d\left(x_{n}, x_{n+1}\right)\right)\left(d\left(x_{n+2}, x_{n+1}\right)\right. \\
& +\phi_{4}\left(d\left(x_{n}, x_{n+1}\right)\right)\left(d\left(x_{n}, x_{n+1}\right)\right)+\phi_{4}\left(d\left(x_{n}, x_{n+1}\right)\right)\left(d\left(x_{n+2}, x_{n+1}\right)\right. \\
& +\phi_{5}\left(d\left(x_{n}, x_{n+1}\right)\right)\left(d\left(x_{n+2}, x_{n+1}\right)\right)+\phi_{6}\left(d\left(x_{n}, x_{n+1}\right)\right)\left(d\left(x_{n}, x_{n+1}\right)\right) \\
& +\phi_{7}\left(d\left(x_{n}, x_{n+1}\right)\right)\left(d\left(x_{n+2}, x_{n+1}\right)\right. \\
& =\left[\phi_{1}\left(d\left(x_{n}, x_{n+1}\right)\right)+\phi_{3}\left(d\left(x_{n}, x_{n+1}\right)\right)+\phi_{4}\left(d\left(x_{n}, x_{n+1}\right)\right)\right.
\end{aligned}
$$

$$
\begin{aligned}
& \left.+\phi_{6}\left(d\left(x_{n}, x_{n+1}\right)\right)\right]\left(d\left(x_{n}, x_{n+1}\right)\right) \\
& +\left[\phi_{2}\left(d\left(x_{n}, x_{n+1}\right)\right)+\phi_{3}\left(d\left(x_{n}, x_{n+1}\right)\right)+\phi_{4}\left(d\left(x_{n}, x_{n+1}\right)\right)\right. \\
& \left.+\phi_{5}\left(d\left(x_{n}, x_{n+1}\right)\right)\left(d\left(x_{n+2}, x_{n+1}\right)\right)+\phi_{7}\left(d\left(x_{n}, x_{n+1}\right)\right)\right]\left(d\left(x_{n+2}, x_{n+1}\right)\right. \\
& =\phi^{\prime}\left(d\left(x_{n}, x_{n+1}\right)\right)\left(d\left(x_{n}, x_{n+1}\right)\right)+\phi^{\prime \prime}\left(d\left(x_{n}, x_{n+1}\right)\right)\left(d\left(x_{n+2}, x_{n+1}\right)\right)
\end{aligned}
$$

then by (5) and definition 2.3, we get

$$
\begin{aligned}
& F\left(d\left(x_{n+1}, x_{n+2}\right)\right) \\
& \quad \leq F\left(\phi^{\prime}\left(d\left(x_{n}, x_{n+1}\right)\right)\left(d\left(x_{n}, x_{n+1}\right)\right)+\phi^{\prime \prime}\left(d\left(x_{n}, x_{n+1}\right)\right)\left(d\left(x_{n+2}, x_{n+1}\right)\right)\right) \\
& \quad-\psi\left(\phi^{\prime}\left(d\left(x_{n}, x_{n+1}\right)\right)\left(d\left(x_{n}, x_{n+1}\right)\right)+\phi^{\prime \prime}\left(d\left(x_{n}, x_{n+1}\right)\right)\left(d\left(x_{n+2}, x_{n+1}\right)\right)\right) .
\end{aligned}
$$

On contrary, if $d\left(x_{n+1}, x_{n+2}\right)>d\left(x_{n}, x_{n+1}\right)$, then

$$
\begin{array}{r}
\phi^{\prime}\left(d\left(x_{n}, x_{n+1}\right)\right)\left(d\left(x_{n}, x_{n+1}\right)\right) \\
+\phi^{\prime \prime}\left(d\left(x_{n}, x_{n+1}\right)\right)\left(d\left(x_{n+2}, x_{n+1}\right)\right)<d\left(x_{n+1}, x_{n+2}\right)
\end{array}
$$

and therefore (6) becomes

$$
F\left(d\left(x_{n+1}, x_{n+2}\right)\right) \leq F\left(d\left(x_{n+1}, x_{n+2}\right)\right)-\psi\left(d\left(x_{n+1}, x_{n+2}\right)\right) .
$$

But, from (3) and the fact that $\psi\left(d\left(x_{n+1}, x_{n+2}\right)\right)>0$, this is a contradiction. Thus, we conclude that

$$
\begin{aligned}
F\left(d\left(x_{n+1}, x_{n+2}\right)\right) & \leq F\left(d\left(x_{n}, x_{n+1}\right)\right)-\psi\left(d\left(x_{n}, x_{n+1}\right)\right) \\
& <F\left(d\left(x_{n}, x_{n+1}\right)\right) .
\end{aligned}
$$

By (7) and Definition 2.1(F1), we have that

$$
d\left(x_{n+1}, x_{n+2}\right)<d\left(x_{n}, x_{n+1}\right)<d\left(x_{n-1}, x_{n}\right) \forall n \in N .
$$

Therefore $\left\{d\left(x_{n}, x_{n+1}\right)\right\}$ is a nonnegative decreasing sequence of real numbers. Thus there exists $\gamma \geq 0$ such that $\lim _{n \rightarrow \infty} d\left(x_{n}, x_{n+1}\right)=$ $\gamma$. From (7) as $n \rightarrow \infty$, we have that

$$
F(\gamma) \leq F(\gamma)-\psi(\gamma)
$$

This implies that $\psi(\gamma)=0$ and thus $\gamma=0$. Consequently we arrive at

$$
\lim _{n \rightarrow \infty} d\left(x_{n}, T x_{n}\right)=\lim _{n \rightarrow \infty} d\left(x_{n}, x_{n+1}\right)=0 .
$$

Now, we claim that $\left\{x_{n}\right\}_{n=1}^{\infty}$ is a Cauchy sequence. On contrary, we assume that there exists $\epsilon>0$ and $n, m \in N$ such that, for all $n \geq n_{\epsilon}$ and $n_{\epsilon}<n<m$,

$$
d\left(x_{n}, x_{m}\right) \geq \epsilon \text { and } d\left(x_{n-1}, x_{m}\right)<\epsilon .
$$

It implies that

$$
\begin{aligned}
\epsilon & \leq d\left(x_{n}, x_{m}\right) \leq d\left(x_{n}, x_{n-1}\right)+s d\left(x_{n-1}, x_{m}\right) \\
& <d\left(x_{n}, x_{n-1}\right)+s \epsilon .
\end{aligned}
$$

By (11) and (9), we have that

$$
\epsilon \leq \underset{n \rightarrow \infty}{\operatorname{limsupd}}\left(x_{n}, x_{m}\right)<s \epsilon .
$$


By triangle inequality, we have that

$$
\begin{aligned}
\epsilon & \leq d\left(x_{n}, x_{m}\right) \leq d\left(x_{n}, x_{m+1}\right)+s d\left(x_{m+1}, x_{m}\right) \\
& \leq d\left(x_{n}, x_{m}\right)+2 s d\left(x_{m+1}, x_{m}\right) .
\end{aligned}
$$

By (9),(10), (12) and (13), we have that

$$
\epsilon \leq \underset{n \rightarrow \infty}{\operatorname{limsupd}}\left(x_{n}, x_{m+1}\right)<s \epsilon .
$$

Similarly, we have that

$$
\begin{aligned}
\epsilon & \leq d\left(x_{n}, x_{m}\right) \leq d\left(x_{n}, x_{n+1}\right)+s d\left(x_{n+1}, x_{m}\right) \\
& \leq s d\left(x_{n}, x_{m}\right)+\left(s^{2}+1\right) d\left(x_{n}, x_{n+1}\right) .
\end{aligned}
$$

By (9),(10), (12) and (15), we have that

$$
\epsilon \leq \operatorname{limsupd}_{n \rightarrow \infty}\left(x_{n}, x_{n+1}\right)<s \epsilon .
$$

Observe that

$$
\begin{aligned}
d\left(x_{n}, x_{m+1}\right) & \leq d\left(x_{n}, x_{n+1}\right)+s d\left(x_{n+1}, x_{m+1}\right) \\
& \leq d\left(x_{n}, x_{n+1}\right)+s\left[d\left(x_{n+1}, x_{m}\right)+s d\left(x_{m+1}, x_{m}\right)\right] \\
& \leq d\left(x_{n}, x_{n+1}\right)+s\left[d\left(x_{n}, x_{n+1}\right)+s d\left(x_{n}, x_{m}\right)\right. \\
& \left.+s d\left(x_{m+1}, x_{m}\right)\right] .
\end{aligned}
$$

By (17), we have that

$$
\frac{\epsilon}{s} \leq \operatorname{limsupd}_{n \rightarrow \infty}\left(x_{n+1}, x_{m+1}\right)<s^{2} \epsilon .
$$

By (9)and (10), we select $n_{\epsilon}>0 \in N$ such that

$$
\begin{aligned}
\frac{1}{s+1} d\left(x_{n}, T x_{n}\right) & <\frac{1}{s+1} \epsilon<\epsilon \leq d\left(x_{n}, x_{m}\right) \forall n \geq n(\epsilon) \\
& \Leftrightarrow \frac{1}{s+1} d\left(x_{n}, T x_{n}\right)<\frac{1}{s+1} \epsilon<d\left(x_{n}, x_{m}\right) \\
\forall n & \geq n(\epsilon) \\
\text { and } & \\
\frac{1}{s+1} d\left(x_{n+1}, S x_{n+1}\right) & <\frac{1}{s+1} \epsilon<\frac{\epsilon}{s} \leq d\left(x_{n+1}, x_{m+1}\right) \forall n \geq n_{\epsilon} \\
& \Leftrightarrow \frac{1}{s+1} d\left(x_{n+1}, S x_{n+1}\right)<\frac{1}{s+1} \epsilon \\
& <d\left(x_{n+1}, x_{m+1}\right) \forall n \geq n_{\epsilon}
\end{aligned}
$$

It follows that from Definition 2.3, we have, for every $n \geq n_{\epsilon}$

$$
F\left(H\left(x_{n+1}, x_{m+1}\right)\right) \leq F\left(N_{\phi}\left(x_{n}, x_{m}\right)\right)-\psi\left(N_{\phi}\left(x_{n}, x_{m}\right)\right) .
$$

Since that

$$
\begin{aligned}
& d\left(x_{n}, x_{m}\right) \leq N_{\phi}\left(x_{n}, x_{m}\right) \\
& =\phi_{1}\left(d\left(x_{n}, x_{m}\right)\right)\left(d\left(x_{n}, x_{m}\right)\right)+\phi_{2}\left(d\left(x_{n}, x_{m}\right)\right)\left(d\left(x_{n+2}, x_{m}\right)\right) \\
& +\phi_{3}\left(d\left(x_{n}, x_{m}\right)\right)\left(\frac{d\left(x_{n+1}, x_{m}\right)+d\left(x_{n}, x_{m+1}\right)}{2 s}\right) \\
& +\phi_{4}\left(d\left(x_{n}, x_{m}\right)\right)\left(\frac{\left(d\left(x_{n+2}, x_{n}\right)+d\left(x_{n+2}, x_{m+1}\right)\right)}{2 s}\right) \\
& +\phi_{5}\left(d\left(x_{n}, x_{m}\right)\right)\left(d\left(x_{n+2}, x_{m+1}\right)+d\left(x_{n+2}, x_{n+1}\right)\right)
\end{aligned}
$$

$$
\begin{aligned}
& +\phi_{6}\left(d\left(x_{n}, x_{m}\right)\right)\left(d\left(x_{n+2}, x_{m+1}\right)+d\left(x_{n}, x_{n+1}\right)\right) \\
& +\phi_{7}\left(d\left(x_{n}, x_{m}\right)\right)\left(d\left(x_{m}, x_{n+1}+d\left(x_{m}, x_{m+1}\right)\right)\right) \\
& \leq \phi_{1}\left(d\left(x_{n}, x_{m}\right)\right)\left(d\left(x_{n}, x_{m}\right)\right)+\phi_{2}\left(d\left(x_{n}, x_{m}\right)\right)\left(d\left(x_{n+2}, x_{n+1}\right)+s d\left(x_{n+1}, x_{m}\right)\right) \\
& +\phi_{3}\left(d\left(x_{n}, x_{m}\right)\right)\left(\frac{d\left(x_{n+1}, x_{m}\right)+d\left(x_{n}, x_{m+1}\right)}{2 s}\right) \\
& +\phi_{4}\left(d\left(x_{n}, x_{m}\right)\right)\left(\frac{\left.\left(d\left(x_{n+2}, x_{n+1}\right)+s d\left(x_{n+1}, x_{n}\right)+d\left(x_{n+2}, x_{n+1}\right)\right)+s d\left(x_{n+1}, x_{m+1}\right)\right)}{2 s}\right) \\
& +\phi_{5}\left(d\left(x_{n}, x_{m}\right)\right)\left(d\left(x_{n+2}, x_{n+1}\right)+s d\left(x_{n+1}, x_{m+1}\right)+d\left(x_{n+2}, x_{n+1}\right)\right) \\
& +\phi_{6}\left(d\left(x_{n}, x_{m}\right)\right)\left(d\left(x_{n+2}, x_{n+1}\right)+s d\left(x_{n+1}, x_{m+1}\right)+d\left(x_{n}, x_{n+1}\right)\right) \\
& \left.+\phi_{7}\left(d\left(x_{n}, x_{m}\right)\right)\left(d\left(x_{m}, x_{n+1}\right)+d\left(x_{m}, x_{m+1}\right)\right)\right) . \\
& \text { By }(12),(14),(16),(18) \text { and }(20), \text { we have that } \\
& \begin{array}{l}
\text { limsupd } \\
\quad n \rightarrow \infty
\end{array} \\
& \left.\left.\quad+x_{n}, x_{m}\right) \leq \limsup _{\phi}(\epsilon)(\epsilon)+\phi_{4}, x_{m}\right)<\phi_{1}(\epsilon)(s \epsilon)+\phi_{2}(\epsilon)\left(s^{2} \epsilon\right) \\
& \quad \leq \max \left\{s \epsilon, s^{2} \epsilon, \epsilon, \frac{s^{2}}{2}, s^{3} \epsilon, s \epsilon\right\} \\
& \quad=s^{3} \epsilon
\end{aligned}
$$

and therefore

$$
\epsilon \leq \underset{n \rightarrow \infty}{\limsup _{\phi}\left(x_{n}, x_{m}\right)<s^{3} \epsilon}
$$

Similarly

$$
\epsilon \leq \liminf _{n \rightarrow \infty} N_{\phi}\left(x_{n}, x_{m}\right)<s^{3} \epsilon
$$

By (19), (21) and (22), we have that

$$
\begin{aligned}
F\left(s^{3} \epsilon\right) & =F\left(s^{4} \frac{\epsilon}{s}\right) \leq F\left(s^{4} \operatorname{limsupd}_{n \rightarrow \infty}\left(x_{n+1}, x_{m+1}\right)\right) \\
& \leq F\left(\underset{n \rightarrow \infty}{\limsup } N_{\phi}\left(x_{n}, x_{m}\right)\right)-\psi\left(\limsup _{n \rightarrow \infty}\left(x_{n}, x_{m}\right)\right) \\
& \leq F\left(s^{3} \epsilon\right)-\psi(\epsilon) .
\end{aligned}
$$

By (23) and the fact that $\epsilon>0$, this is a contradiction. Hence $\left\{x_{n}\right\}$ is a Cauchy sequence in $X$. By completeness of $(X, d)$, $\left\{x_{n}\right\}_{n=1}^{\infty}$ and $\left\{x_{n+1}\right\}_{n=1}^{\infty}$ converge to some point $x^{*} \in X$, that is,

$$
\lim _{n \rightarrow \infty} d\left(x_{n}, x^{*}\right)=0 \text { and } \lim _{n \rightarrow \infty} d\left(x_{n+1}, x^{*}\right)=0 .
$$

There exists increasing sequences $\left\{n_{k}\right\},\left\{n+1_{k}\right\} \subset N$ such that $x_{n_{k}} \in T x^{*}$ and $x_{n+1_{k}} \in S x^{*}$ for all $k \in N$. Since $T x^{*}$ and $S x^{*}$ are closed and

$$
\lim _{n \rightarrow \infty} d\left(x_{n_{k}}, x^{*}\right)=0 \text { and } \lim _{n \rightarrow \infty} d\left(x_{n+1_{k}}, x^{*}\right)=0,
$$

we get $x^{*} \in T x^{*}$ and $x^{*} \in S x^{*}$.

Step two. We show that $x^{*}$ is a common fixed point of $T$ and $S$. It suffices to show that

$$
\begin{aligned}
& \frac{1}{1+s} d\left(x_{n}, T x_{n}\right)<d\left(x_{n}, x^{*}\right) \text { and } \frac{1}{1+s} d\left(x_{n+1}, S x_{n+1}\right)<d\left(x_{n+1}, x^{*}\right) \\
& \text { for every } n \in N
\end{aligned}
$$


implies

$$
\left.d\left(T x^{*}, x^{*}\right)+d\left(x^{*}, x_{n+1}\right)\right\}
$$

$$
F\left(d\left(T x^{*}, x^{*}\right)\right) \leq F\left(N_{\phi}\left(x^{*}, T x^{*}\right)\right)-\psi\left(N_{\phi}\left(x^{*}, T x^{*}\right)\right)
$$

and

$$
F\left(d\left(S x^{*}, x^{*}\right)\right) \leq F\left(N_{\phi}\left(S x^{*}, x^{*}\right)\right)-\psi\left(N_{\phi}\left(S x^{*}, x^{*}\right)\right),
$$

respectively.

On contrary, suppose there exists $m \in N$ such that

$\frac{1}{1+s} d\left(x_{m}, T x_{m}\right) \geq d\left(x_{m}, x^{*}\right)$ or $\frac{1}{1+s} d\left(x_{m+1}, S x_{m+1}\right) \geq d\left(x_{m+1}, x^{*}\right)$.

By (26), we have that

$$
(s+1) d\left(x_{m}, x^{*}\right) \leq d\left(x_{m}, T x_{m}\right) \leq d\left(x_{m}, x^{*}\right)+s d\left(T x_{m}, x^{*}\right)
$$

or

$(s+1) d\left(x_{m+1}, x^{*}\right) \leq d\left(x_{m+1}, S x_{m+1}\right) \leq d\left(x_{m+1}, x^{*}\right)+s d\left(S x_{m+1}, x^{*}\right)$, and therefore

$$
\begin{aligned}
& d\left(x_{m}, x^{*}\right) \leq d\left(T x_{m}, x^{*}\right)=d\left(x_{m+1}, x^{*}\right) \text { and } \\
& d\left(x_{m+1}, x^{*}\right) \leq d\left(S x_{m+1}, x^{*}\right)=d\left(x_{m+2}, x^{*}\right) .
\end{aligned}
$$

By (8), (26) and (27), this is a contradiction. Hence, (25) holds, and therefore

$$
\begin{aligned}
F\left(d\left(x_{n+1}, x^{*}\right)\right) & =F\left(H\left(T x_{n}, S x^{*}\right)\right) \\
& \leq F\left(N_{\phi}\left(x_{n}, x^{*}\right)\right)-\psi\left(N_{\phi}\left(x_{n}, x^{*}\right)\right),
\end{aligned}
$$

and

$$
\begin{aligned}
F\left(d\left(x_{n+2}, x^{*}\right)\right) & =F\left(H\left(S x_{n+1}, T x^{*}\right)\right) \\
& \leq F\left(N_{\phi}\left(x_{n+1}, x^{*}\right)\right)-\psi\left(N_{\phi}\left(x_{n+1}, x^{*}\right)\right) .
\end{aligned}
$$

Since that

$$
\begin{aligned}
d\left(x^{*}, T x^{*}\right) & \leq N_{\phi}\left(x_{n}, x^{*}\right) \\
& =\phi_{1}\left(d\left(x_{n}, x^{*}\right)\right)\left(d\left(x_{n}, x^{*}\right)\right)+\phi_{2}\left(d\left(x_{n}, x^{*}\right)\right)\left(d\left(x_{n+2}, x^{*}\right)\right) \\
& +\phi_{3}\left(d\left(x_{n}, x^{*}\right)\right)\left(\frac{d\left(x_{n+1}, x^{*}\right)+d\left(x_{n}, S x^{*}\right)}{2 s}\right) \\
& +\phi_{4}\left(d\left(x_{n}, x^{*}\right)\right)\left(\frac{d\left(x_{n}, S x^{*}\right)+d\left(S x^{*}, x_{n+2}\right)}{2 s}\right) \\
& +\phi_{5}\left(d\left(x_{n}, x^{*}\right)\right)\left(d\left(S x^{*}, x_{n+2}\right)+d\left(x_{n+1}, S x^{*}\right)\right) \\
& +\phi_{6}\left(d\left(x_{n}, x^{*}\right)\right)\left(d\left(x_{n}, x_{n+1}\right)+d\left(x_{n+2}, T x^{*}\right)\right) \\
& +\phi_{7}\left(d\left(x_{n}, x^{*}\right)\right)\left(d\left(T x^{*}, x^{*}\right)+d\left(x^{*}, x_{n+1}\right)\right) \\
& \leq \max _{\{}\left(d\left(x_{n}, x^{*}\right), d\left(x_{n+2}, x^{*}\right),\right. \\
& \frac{d\left(x_{n+1}, x^{*}\right)+d\left(x_{n}, S x^{*}\right)}{2 s}, \\
& \frac{d\left(x_{n}, S x^{*}\right)+s d\left(S x^{*}, x_{n+2}\right)+d\left(S x^{*}, x_{n+2}\right)}{2 s}, \\
& d\left(S x^{*}, x_{n+2}\right)+d\left(x_{n+1}, S x^{*}\right), d\left(x_{n}, x_{n+1}\right)+d\left(x_{n+2}, T x^{*}\right),
\end{aligned}
$$

and

$$
\begin{aligned}
d\left(x^{*}, S x^{*}\right) & \leq N_{\phi}\left(x_{n+1}, x^{*}\right) \\
& =\phi_{1}\left(d\left(x_{n+1}, x^{*}\right)\right)\left(d\left(x_{n+1}, x^{*}\right)\right)+\phi_{2}\left(d\left(x_{n+1}, x^{*}\right)\right)\left(d\left(x^{*}, x_{n+3}\right)\right) \\
& \left.+\phi_{3}\left(x_{n+1}, x^{*}\right)\right)\left(\frac{d\left(x_{n+2}, x^{*}\right)+d\left(x_{n+1}, x^{*}\right)}{2 s}\right) \\
& +\phi_{4}\left(d\left(x_{n+1}, x^{*}\right)\right)\left(\frac{d\left(x_{n+1}, S x^{*}\right)+d\left(S x^{*}, x_{n+3}\right)}{2 s}\right) \\
& +\phi_{5}\left(d\left(x_{n+1}, x^{*}\right)\right)\left(d\left(x_{n+3}, S x^{*}\right)+d\left(x_{n+2}, S x^{*}\right)\right) \\
& +\phi_{6}\left(d\left(x_{n+1}, x^{*}\right)\right)\left(d\left(x_{n+1}, x_{n+2}\right)+d\left(x_{n+3}, S x^{*}\right)\right) \\
& +\phi_{7}\left(d\left(x_{n+1}, x^{*}\right)\right)\left(d\left(S x^{*}, x^{*}\right)+d\left(x^{*}, x_{n+2}\right)\right) \\
& \leq \max \left\{d\left(x_{n+1}, x^{*}\right), d\left(x^{*}, x_{n+3}\right)\right. \\
& \frac{d\left(x_{n+2}, x^{*}\right)+d\left(x_{n+1}, x^{*}\right)}{2 s}, \\
& \frac{d\left(x_{n+1}, x_{n+2}\right)+s d\left(x_{n+2}, S x^{*}\right)+d\left(S x^{*}, x_{n+3}\right)}{2 s} \\
& d\left(x_{n+3}, S x^{*}\right)+d\left(x_{n+2}, S x^{*}\right), d\left(x_{n+1}, x_{n+2}\right)+d\left(x_{n+3}, S x^{*}\right) \\
& \left.d\left(S x^{*}, x^{*}\right)+d\left(x^{*}, x_{n+2}\right)\right\} .
\end{aligned}
$$

By (24) and (30), we have that

$$
\lim _{n \rightarrow \infty} N_{\phi}\left(x_{n}, x^{*}\right)=d\left(T x^{*}, x^{*}\right) .
$$

By (24) and (31), we have that

$$
\lim _{n \rightarrow \infty} N_{\phi}\left(x_{n+1}, x^{*}\right)=d\left(S x^{*}, x^{*}\right) .
$$

By (28)and (29) and by the continuity of $F$ and $\psi$, we have that

$$
F\left(d\left(x^{*}, T x^{*}\right)\right) \leq F\left(N_{\phi}\left(x^{*}, T x^{*}\right)\right)-\psi\left(N_{\phi}\left(x^{*}, T x^{*}\right)\right),
$$

and

$$
F\left(d\left(x^{*}, S x^{*}\right)\right) \leq F\left(N_{\phi}\left(x^{*}, S x^{*}\right)\right)-\psi\left(N_{\phi}\left(x^{*}, S x^{*}\right)\right) .
$$

Hence, since $T x^{*}$ and $S x^{*}$ are closed then we have $x^{*} \in T x^{*}$ and $x^{*} \in S x^{*}$, that is, $x^{*}$ is a fixed point of $T$ and $S$.

In Theorem 2.1, when $T=S=U$, then we have the following result.

Corollary 2.1.1. Let $(X, d)$ be a complete strong b-metric space and let $U: X \rightarrow C B(X)$ be a multivalued generalized $F$-SuzukiContraction mapping. Then $U$ has a fixed point $x^{*} \in X$ and for every $x \in X$ the sequence $\left\{U^{n} x\right\}_{n=1}^{\infty}$ converges to $x^{*}$.

In Corollary 2.1.1, when $U$ is a single-valued then we have another new result as follows.

Corollary 2.1.2. Let $(X, d)$ be a complete strong $b$-metric space and let $U: X \rightarrow X$ be a single-valued generalized F-SuzukiContraction mapping. Then $U$ has a fixed point $x^{*} \in X$ and for every $x \in X$ the sequence $\left\{U^{n} x\right\}_{n=1}^{\infty}$ converges to $x^{*}$.

In Theorem 2.1, when $T$ and $S$ are two single-valued then the 
following result holds.

Corollary 2.1.3. Let $(X, d)$ be a complete strong $b$-metric space and let $T, S: X \rightarrow X$ be two single-valued generalized $F$ Suzuki-Contraction mappings. Then $T$ and $S$ have a common fixed point $x^{*} \in X$ and for every $x \in X$ the sequence $\left\{T^{n} x\right\}_{n=1}^{\infty}$ and $\left\{S^{n} x\right\}_{n=1}^{\infty}$ converge to $x^{*}$.

In Theorem 2.1, when $(X, d)$ is a complete $b$-metric space then the following new result holds.

Corollary 2.1.4. Let $(X, d)$ be a complete $b$-metric space and let $T, S: X \rightarrow X$ be two single-valued generalized $F$-SuzukiContraction mappings. Then $T$ and $S$ have a common fixed point $x^{*} \in X$ and for every $x \in X$ the sequence $\left\{T^{n} x\right\}_{n=1}^{\infty}$ and $\left\{S^{n} x\right\}_{n=1}^{\infty}$ converge to $x^{*}$.

In corollary 2.1.4, when $T=S=U$, then we have the following result.

Corollary 2.1.5. Let $(X, d)$ be a complete $b$-metric space and let $U: X \rightarrow C B(X)$ be a multivalued generalized F-SuzukiContraction mapping. Then $U$ has a fixed point $x^{*} \in X$ and for every $x \in X$ the sequence $\left\{U^{n} x\right\}_{n=1}^{\infty}$ converges to $x^{*}$.

Corollary 2.1.6. Let $(X, d)$ be a complete strong $b$-metric space and let $U: X \rightarrow C B(X)$ be a multivalued generalized $F$ Suzuki-Contraction mapping such that there exists $F \in \mho$ and $\psi \in \Psi, \forall x, y \in X, x \neq y, \frac{1}{s+1} d(x, U x)<d(x, y) \Rightarrow \psi(N(x, y))+$ $F\left(s^{4} d(U x, U y)\right) \leq F(N(x, y))$ in which

$$
\begin{aligned}
& N(x, y)=\max \left\{d(x, y), d\left(y, U^{2} x\right),\right. \\
& \frac{(d(y, U x))+d(x, U y)}{2 s}, \frac{(d(x, U y))+d\left(U^{2} x, U y\right)}{2 s}, \\
& d\left(U^{2} x, U y\right)+d(U y, U x), d\left(U^{2} x, U y\right)+d(U x, x), \\
& d(U x, y))+d(y, U y)\} .
\end{aligned}
$$

Then $U$ has a fixed point $x^{*} \in X$ and for every $x \in X$ the sequence $\left\{U^{n} x\right\}_{n=1}^{\infty}$ converges to $x^{*}$.

Proof from Lemma 1.1, since (2) $\Rightarrow$ (32) then by the corollary 2.1.1 the result follows immediately.

Corollary 2.1.7. Let $(X, d)$ be a complete strong $b$-metric space and let $U: X \rightarrow X$ be a single-valued generalized F-SuzukiContraction mapping such that there exists $F \in \mho$ and $\psi \in$ $\Psi, \forall x, y \in X, x \neq y, \frac{1}{s+1} d(x, U x)<d(x, y) \Rightarrow \psi(N(x, y))+$ $F\left(s^{4} d(U x, U y)\right) \leq F(N(x, y))$ in which

$$
\begin{aligned}
& N(x, y)=\max \left\{d(x, y), d\left(y, U^{2} x\right),\right. \\
& \frac{(d(y, U x))+d(x, U y)}{2 s}, \frac{(d(x, U y))+d\left(U^{2} x, U y\right)}{2 s}, \\
& d\left(U^{2} x, U y\right)+d(U y, U x), d\left(U^{2} x, U y\right)+d(U x, x), \\
& d(U x, y))+d(y, U y)\} .
\end{aligned}
$$

Then $U$ has a fixed point $x^{*} \in X$ and for every $x \in X$ the sequence $\left\{U^{n} x\right\}_{n=1}^{\infty}$ converges to $x^{*}$.

Proof from Lemma 1.1, since (2) $\Rightarrow$ (33) then by the corollary 2.1.2 the result holds.
Corollary 2.1.8. Let $(X, d)$ be a complete strong $b$-metric space and let $T, S: X \rightarrow X$ be two single-valued generalized $F$ Suzuki-Contraction mappings such that there exists $F \in \mho$ and $\psi \in \Psi, \forall x, y \in X, x \neq y, \frac{1}{s+1} d(x, T x)<d(x, y)$ and $\frac{1}{s+1} d(y, S x)<$ $d(y, S T x) \Rightarrow \psi(N(x, y))+F\left(s^{4} H(T x, S y)\right) \leq F(N(x, y))$ in which

$N(x, y)=\max \{d(x, y), d(y, S T x)$,

$\frac{(d(y, T x))+d(x, S y)}{2 s}, \frac{(d(x, S y))+d(S T x, S y)}{2 s}$,

$d(S T x, S y)+d(S y, T x), d(S T x, S y)+d(T x, x), d(T x, y))+d(y, S y)\}$.

Then $T$ and $S$ have a common fixed point $x^{*} \in X$ and for every $x \in X$ the sequence $\left\{T^{n} x\right\}_{n=1}^{\infty}$ and $\left\{S^{n} x\right\}_{n=1}^{\infty}$ converge to $x^{*}$.

Proof from Lemma 1.1 , since $(2) \Rightarrow(34)$ then by the corollary 2.1.4 the result holds.

Corollary 2.1.9. Let $(X, d)$ be a complete $b$-metric space and let $U: X \rightarrow C B(X)$ be a multivalued generalized F-SuzukiContraction mapping such that there exists $F \in \mho$ and $\psi \in$ $\Psi, \forall x, y \in X, x \neq y, \frac{1}{2 s} d(x, U x)<d(x, y) \Rightarrow \psi(N(x, y))+$ $F\left(s^{6} d(U x, U y)\right) \leq F(N(x, y))$ in which

$N(x, y)=\max \left\{d(x, y), d\left(y, U^{2} x\right)\right.$,

$\frac{(d(y, U x))+d(x, U y)}{2 s}, \frac{(d(x, U y))+d\left(U^{2} x, U y\right)}{2 s}$,

$\left.\left.d\left(U^{2} x, U y\right)+d(U y, U x), d\left(U^{2} x, U y\right)+d(U x, x), d(U x, y)\right)+d(y, U y)\right\}$.

Then $U$ has a fixed point $x^{*} \in X$ and for every $x \in X$ the sequence $\left\{U^{n} x\right\}_{n=1}^{\infty}$ converges to $x^{*}$.

Proof from Lemma 1.1, since (2) $\Rightarrow$ (35) then by the corollary 2.1.5 the result holds.

\section{Example}

Let $X=[0,1] . T, S:[0,1] \rightarrow C B([0,1])$ be defined by $T x=$ $\left[0, \frac{x}{2}\right]$ and $S y=\left[0, \frac{y}{2}\right]$ such that $S T x=\left[0, \frac{x}{8}\right]$ for all $x \in[0,1]$. Let $d$ be the usual metric on $X$. Taking $F(t)=\frac{t}{10}$ and let $x<y$, then $\forall x, y \in[0,1] d(x, y)>0$ and $d(y, S T x)=\left|y-\frac{x}{8}\right|>\left|y-\frac{y}{8}\right|=$ $\frac{7}{8} y>\frac{y}{4}$. Now, for $s=1$, we have that $\frac{1}{2} d(x, T x)=0<d(x, y)$ and $\frac{1}{2} d(y, S y)=\frac{y}{4}<d(y, S T x)$. Without lose of generality, let $\phi_{1}(d(x, y))=\phi_{2}(d(x, y))=\phi_{3}(d(x, y))=\frac{1}{5}$; and $\phi_{4}(d(x, y))=$ $\phi_{5}(d(x, y))=\phi_{6}(d(x, y))=\phi_{7}(d(x, y))=\frac{1}{10^{2}}$. Therefore, we have that

$$
\begin{aligned}
& F(H(T x, S y))=\ln (H(T x, S y))+H(T x, S y) \\
& =\frac{1}{10}\left|\frac{y}{2}-\frac{x}{4}\right|=\frac{1}{10}\left|y-\frac{y}{2}-\frac{x}{4}\right| \\
& \leq \frac{1}{10}\left(\left|y-\frac{x}{4}\right|+\left|x-\frac{y}{2}\right|\right) \\
& =\frac{1}{10}\left(\frac{\left|y-\frac{x}{4}\right|+\left|x-\frac{y}{2}\right|}{2}\right)+\frac{1}{10}\left(\frac{\left|y-\frac{x}{4}\right|+\left|x-\frac{y}{2}\right|}{2}\right)
\end{aligned}
$$




$$
\begin{aligned}
& \leq \frac{1}{10}\left(\frac{\left|y-\frac{x}{4}\right|+\left|x-\frac{y}{2}\right|}{2}\right)+\frac{1}{10}\left(\frac{\left|y-\frac{x}{4}\right|+\left|x-\frac{x}{8}\right|+\left|\frac{x}{8}-\frac{y}{2}\right|}{2}\right) \\
& =\frac{1}{10}\left(\frac{\left|y-\frac{x}{4}\right|+\left|x-\frac{y}{2}\right|}{2}\right)+\frac{1}{10}\left(\frac{\left|\frac{x}{8}-\frac{y}{2}\right|+\left|x-\frac{x}{8}\right|}{2}\right) \\
& +\frac{1}{10}\left(\left|\frac{y}{2}-\frac{x}{8}\right|\right) \leq \frac{1}{10}\left(\frac{\left|y-\frac{x}{4}\right|+\left|x-\frac{y}{2}\right|}{2}\right) \\
& +\frac{1}{10}\left(\frac{\left|\frac{x}{8}-\frac{y}{2}\right|+\left|x-\frac{x}{8}\right|}{2}\right)+\frac{1}{10}\left(\left|\frac{y}{2}-\frac{x}{8}\right|+\left|\frac{x}{8}-\frac{x}{4}\right|\right) \\
& =\frac{1}{5}\left(\frac{\left|y-\frac{x}{4}\right|+\left|x-\frac{y}{2}\right|}{2}\right)+\frac{1}{5}\left(\frac{\left|\frac{x}{8}-\frac{y}{2}\right|+\left|x-\frac{x}{8}\right|}{2}\right) \\
& +\frac{1}{10}\left(\left|\frac{y}{2}-\frac{x}{8}\right|+\left|\frac{x}{8}-\frac{x}{4}\right|\right)+\frac{1}{10^{2}}(|x-y|)+\frac{1}{10^{2}}\left(\left|y-\frac{x}{8}\right|\right) \\
& +\frac{1}{10^{2}}\left(\left|\frac{x}{8}-\frac{y}{2}\right|+\left|\frac{x}{4}-x\right|\right)+\frac{1}{10^{2}}\left(\left|\frac{x}{4}-y\right|+\left|y-\frac{y}{2}\right|\right) \\
& -\frac{1}{10^{2}}\left[(|x-y|)+\left(\left|y-\frac{x}{8}\right|\right)+\left(\left|\frac{x}{8}-\frac{y}{2}\right|+\left|\frac{x}{4}-x\right|\right)\right] \\
& +\left(\left|\frac{x}{4}-y\right|+\left|y-\frac{y}{2}\right|\right)-\frac{1}{10}\left(\left|\frac{y}{2}-\frac{x}{8}\right|+\left|\frac{x}{8}-\frac{x}{4}\right|\right) \\
& -\frac{1}{10}\left(\frac{\left|y-\frac{x}{4}\right|+\left|x-\frac{y}{2}\right|}{2}\right)-\frac{1}{10}\left(\frac{\left|\frac{x}{8}-\frac{y}{2}\right|+\left|x-\frac{x}{8}\right|}{2}\right) \text {. } \\
& =\phi_{1}(d(x, y))(d(x, y))+\phi_{2}(d(x, y))(d(y, S T x)) \\
& +\phi_{3}(d(x, y))\left(\frac{(d(y, T x))+d(x, S y)}{2 s}\right)+\phi_{4}(d(x, y)) \\
& \left(\frac{(d(x, S T x))+d(S T x, S y)}{2 s}\right) \\
& +\phi_{5}(d(x, y))(d(S T x, S y)+d(S T x, T x)) \\
& +\phi_{6}(d(x, y))(d(S T x, S y)+d(T x, x)) \\
& \left.+\phi_{7}(d(x, y))(d(T x, y))+d(y, S y)\right)-\psi\left(N_{\phi}(x, y)\right) \text {. }
\end{aligned}
$$

\section{Conclusion}

Fixed point results of Piri and Kumam [11], Ahmad et al. [9], Suzuki [18] and Suzuki [19] are extended by introducing common fixed point problem for multivalued generalized F-Suzukicontraction mappings in strong b-metric spaces. In specific, Corollary 2.1.1 and corollary 2.1.2 generalize and extend the work of Ahmad et al. [9] and Kumam and Hossein [5], respectively.

\section{Acknowledgments}

I thank the referees for the positive enlightening comments and suggestions, which have greatly helped me in making improve- ments to this paper.

\section{References}

[1] B. Banach, "Sur les operations dons les ensembles abstraits et leur application aux equations intégrales", Fundam. Math. 3 (1922) 133.

[2] M. Edelstein, "On fixed and periodic points under contractive mappings", 37 (1962) 74.

[3] D. Wardowski, "Fixed point theory of a new type of contractive mappings in complete metric spaces", Fixed Point Theory Appl. 2012 (2012) 94.

[4] D. Wardowski \& N. V. Dung, "Fixed points of $f$-weak contractions on complete metric spaces", Demonstr. Math. 1 (2014) 146.

[5] P. Kumam, N. V. Dung \& K. Sitthithakerngkiet, "A generalization of Ciric fixed point theorem", Filomat 29 (2015) 1549.

[6] A. K. Dubey, S. Rita \& R. P. Dubey, "Some Fixed Point Results in bmetric Spaces", Asian Journal of Mathematics and Applications 2014 (2014) 2307.

[7] H. H. Alsulami, K. J. Erdal \& P. Hossein, "Fixed Points of Generalized F-Suzuki Type Contraction in Complete b-Metric Spaces", Discrete Dynamics in Nature and Society 2015 (2015) 969726. http://dx.doi.org/10.1155/2015/969726.

[8] D. Wardowski, "Fixed point theory of a new type of contractive mappings in complete metric spaces", Fixed Point Theory Appl. 2012 (2012) 94.

[9] J. Ahmad, A. Al-Rawashdeh \& A. Azam, "New fixed point theorems for generalized F-contractions in complete metric spaces, Fixed Point Theory and Applications. 2015 (2015) 80. https://doi.org/10.1186/s13663-0150333-2

[10] V. D. Nguyen \& T. H. Vo, "A Fixed Point Theorem for Generalized FContractions on Complete Metric Spaces", Vietnam J. Math. 43 (2014) 743. https://doi.org/10.1007/s10013-015-0123-5

[11] H. Piri \& P. Kumam, "Some fixed point theorems concerning Fcontraction in complete metric spaces", Fixed Point Theory Appl. 2014 (2014) 210. https://doi.org/10.1186/1687-1812-2014-210.

[12] H. Piri \& P. Kumam, "Fixed point theorems for generalized F-Suzukicontraction mappings in complete b-metric spaces", Fixed Point Theory and Applications 2016 (2016) 90. https://doi.org/10.1186/s13663-0160577-5.

[13] B. E. Rhoades, "A comparison of various definition of contractive mappings", Trans. Amer. Math. Soc. 226 (1977) 257.

[14] G. E. Hardy \& T. D. Rogers, "A generalization of a fixed point theorem of Reich", Canad. Math. Bull. 16 (1973) 201.

[15] P. Hossein \& P. Kumam, "Some fixed point theorems concerning Fcontraction in complete metric spaces", Fixed point theory and applications 2014 (2014) 210. https://doi.org/10.1186/1687-1812-2014-210

[16] T. Rasham, A. Shoaib, B. A. S. Alamri \& M. Arshad, "Multivalued Fixed Point Results for New Generalized $F$-Dominated Contractive Mappings on Dislocated Metric Space with Application", Journal of Function Spaces Volume 2018 (2018) 4808764. https://doi.org/10.1155/2018/4808764.

[17] T. Rasham, A. Shoaib, N. Hussain, M. Arshad \& S. U. Khan, "Common fixed point results for new Ciric-type rational multivalued F-contraction with an application", J. Fixed Point Theory Appl. 2018 (2018) 20 https://doi.org/10.1007/s11784-018-0525-6.

[18] T. Suzuki, "A generalized Banach contraction principle that characterizes metric completeness", Proceedings of the American Mathematical Society 136 (2008) 1861.

[19] T. Suzuki, "Discussion of several contractions by Jachymski's approach", Fixed point theory and applications 2016 (2016) 91. https://doi.org/10.1186/s13663-016-0581-9 